\title{
A GENERALIZATION OF NONCOMMUTATIVE JORDAN ALGEBRAS
}

\author{
R. W. RITCHIE 1
}

A noncommutative Jordan algebra was defined by R. D. Schafer [7] as an algebra satisfying the Jordan identity

$$
\left(x^{2} y\right) x=x^{2}(y x)
$$

and the flexible law

$$
(x y) x=x(y x) .
$$

In this paper we replace (2) by the weaker ${ }^{2}$ hypothesis

$$
x^{2} x=x x^{2}
$$

and consider algebras satisfying (1) and (3).

In $\S 1$ we show that such algebras of characteristic not 2 or 3 are strictly power-associative, and in $\$ 2$ we establish several properties of the submodules $A_{e}(\lambda)$ of these algebras. In the last section we use these results and follow the arguments in [6] to show the existence of an identity element in the finite dimensional semi-simple case. Then the results of [6] and [7] allow us to obtain the following theorems.

THEOREM 1. Every finite dimensional semi-simple algebra of characteristic not 2 or 3 satisfying (1) and (3) is a noncommutative Jordan algebra with identity element, and thus is the direct sum of simple subalgebras.

THEOREM 2. A finite dimensional simple algebra of characteristic not 2 or 3 satisfying (1) and (3) is either a (commutative) Jordan algebra, a quasi-associative algebra, or an algebra of degree at most 2.

1. Let $A$ be an algebra of characteristic $p$ not 2 or 3 satisfying (1) and (3). Identity (3) yields [1, Lemma 2] and in particular $x^{3} x=x x^{3}$. Since (1) implies $x^{3} x=x^{2} x^{2}$ we have $x^{n-a} x^{a}=x^{n-1} x$ for all $a<n, n=3$ or 4 . Now there are two cases.

CASE $1, p \neq 2,3$ or 5 . We have [1, Lemma 4] so that, assuming $x^{a} x^{b}=x^{a+b}$ for all $a+b<n$ and any $n \geqq 5$, we get

Received by the editors November 6, 1958 and, in revised form, March 4, 1959.

1 National Science Foundation Predoctoral Fellow.

${ }^{2} \mathrm{An}$ algebra satisfying (1) and (3) but not (2) is given by basis $e, u, v, w$ with $e^{2}=e, u e=u, v w=-w v=u$, and all remaining products zero. 


$$
x^{2} x^{n-2}=x^{n-1} x+\frac{1}{2}(n-3)\left[x^{n-1}, x\right]
$$

Now letting $y=x^{n-3}$ in (1) and using (4) yields $(n-3)\left[x^{n-1}, x\right]=0$. Since $p \neq 3$ this gives us $\left[x^{k p-1}, x\right]=0$ for all $k \geqq 1$, and combining this result with $[1$, Lemmas 2 and 4$]$ yields $x^{n-a} x^{a}=x^{n-1} x$ for all $1<a<n$ so that $A$ is power-associative.

CASE $2, p=5$. Since the base field has at least five elements, a substitution of $x+\lambda x^{2}$ for $x$ and of $x$ for $y$ in (1) yields $x^{3} x^{2}+2 x^{4} x=x^{2} x^{3}$ $+2 x^{3} x^{2}$. Letting $y=x^{2}$ in (1) yields $x^{4} x=x^{2} x^{3}$, so, combining these two results, we have $\left[x^{3}, x^{2}\right]=0$. But by $[1$, Lemma 2] we see that this implies $\left[x^{4}, x\right]=0$. We also have that (1) implies $x^{5} x=x^{4} x^{2}$ and [1, Lemma 2] yields $x^{2} x^{4}=x^{4} x^{2}$. Thus the hypotheses of [5, Lemma 4] are satisfied, so that (4) holds in this case also. Then $\left[x^{k-1}, x\right]=0$ by the proof of Case 1 above, and it follows that $A$ is power-associative from the proof above using [5, Lemma 4] in place of [1, Lemma 4].

Cases 1 and 2 above, together with an observation that (1) and (3) are both equivalent to multi-linear identities since $p>3$, complete the proof of the following result.

Lemma 1. An algebra of characteristic not 2 or 3 satisfying (1) and (3) is strictly power-associative.

However, such algebras of characteristic $p=2$ or $p=3$ need not be power-associative. In the case $p=2$ this is easily seen from the algebra constructed in $[1$, p. 25], while for $p=3$ this is shown by the example in $[5, \S 5]$ taking $p=3$ and $n=2$.

2. Throughout this section $A$ will be an algebra of characteristic not 2 or 3 which satisfies (1) and (3). The notations will be those of [6] except that the multiplication $*$ of $A^{+}$is defined by $x * y=x y+y x$, and, further, $e$ will always be an idempotent.

Observing that $e / 2$ is an idempotent of the commutative algebra $A^{+}$, and that $A_{e / 2}^{+}(\lambda)=A_{e}(\lambda)$ for $\lambda=0,1$ and 2 , we are able to state $\left[2\right.$, Theorem 2] in the following form. $A_{e}(2)$ and $A_{e}(0)$ are orthogonal subrings of $A^{+}, A_{e}(1) * A_{e}(1) \subset A_{e}(2)+A_{e}(0)$, and $A_{e}(\lambda) * A_{e}(1)$ $\subset A_{e}(1)+A_{e}(2-\lambda)$ for $\lambda=0$ or 2. In [2], Albert also showed that the submodules $A_{e}(2)$ and $A_{e}(0)$ of $A$ are orthogonal and that $e a_{2}=a_{2} e=a_{2}$ and $e a_{0}=a_{0} e=0$ for all $a_{2} \in A_{e}(2), a_{0} \in A_{e}(0)$. These results are basic to the subsequent development.

Linearization of (1) yields

$$
[x z+z x, y, w]+[x w+w x, y, z]+[z w+w z, y, x]=0
$$


where of course $[x, y, z]=(x y) z-x(y z)$. Letting $w=z=e$ and $x=x_{\lambda}$ in (5) we have

$$
\lambda\left[x_{\lambda}, y, e\right]+\left[e, y, x_{\lambda}\right]=0 .
$$

Lemma 2. $e \cdot A_{e}(1) \subset A_{e}(1)$, and $A_{e}(1) \cdot e \subset A_{e}(1)$.

Proof. Letting $x=e$ and $y=y_{1}$ in (1) we find $\left(e y_{1}\right) e=e\left(y_{1} e\right)$. Thus $e y_{1} * e=e\left(y_{1} e+e y_{1}\right)=e y_{1}$ so that $e y_{1} \in A_{e}(1)$ by definition. Observing that $y_{1} e=e * y_{1}-e y_{1}$ completes the proof.

Lemma 3. $A_{e}(0) \cdot A_{e}(0) \subset A_{e}(0)+A_{e}(1)$.

Proor. Let $y=y_{0}$ and $\lambda=0$ in (6), obtaining $0=\left(e y_{0}\right) x_{0}=e\left(y_{0} x_{0}\right)$. Thus $e\left[y_{0} x_{0}\right]_{2}=\left[y_{0} x_{0}\right]_{2}=0$.

Now we linearize (3) to find

$$
[e, y * z]+[z, e * y]+[y, e * z]=0 .
$$

Noting that $e * x=2[x]_{2}+[x]_{1}$ and letting $y=y_{2}+y_{1}+y_{0}$ and $z=z_{2}+z_{1}+z_{0}$, we compute $[z, e * y]$ and $[y, e * z]$ in terms of components, and substituting in (7) gives

$$
[y * z, e]=\left[z_{1}, y_{2}\right]+\left[y_{1}, z_{2}\right]+\left[z_{0}, y_{1}\right]+\left[y_{0}, z_{1}\right] .
$$

Lemia $4 .^{3} A_{e}(1) \cdot A_{e}(\lambda) \subset A_{e}(1)+A_{e}(2-\lambda)$ and

$$
A_{e}(\lambda) \cdot A_{e}(1) \subset A_{e}(1)+A_{e}(2-\lambda) \text { for } \lambda=0 \text { or } 2 .
$$

Proof. We observe that $[x, e]=\left[[x]_{1}, e\right]$. Now let $z=z_{1}$ and $y=y_{0}$ in (8) to obtain $\left[y_{0} * z_{1}, e\right]=\left[y_{0}, z_{1}\right]$. Therefore $\left[y_{0}, z_{1}\right] \in A_{e}(1)$. But since $A^{+}$is strictly power-associative, $y_{0} * z_{1} \in A_{e}(1)+A_{e}(2)$. Now since $y_{0} z_{1}=2^{-1}\left(y_{0} * z_{1}+\left[y_{0}, z_{1}\right]\right)$ and $z_{1} y_{0}=2^{-1}\left(y_{0} * z_{1}-\left[y_{0}, z_{1}\right]\right)$, both $y_{0} z_{1}$ and $z_{1} y_{0}$ are elements of $A_{e}(1)+A_{e}(2)$. A parallel argument completes the proof of the lemma.

Turning to fourth powers, we find that a linearization of $x^{3} x=x^{2} x^{2}$ yields

$$
\begin{aligned}
& {[(x * e) z+(z * e) x+(z * x) e] e+[(x * e) e+e x] z+[(z * e) e+e z] x } \\
&=(x * e) *(z * e)+(x * z) * e .
\end{aligned}
$$

In the case that $x=x_{0}$ and $z=z_{1}$ this identity becomes

$$
\left(z_{1} x_{0}\right) e+z_{1} x_{0}=\left(x_{0} * z_{1}\right) * e-\left[\left(z_{1} * x_{0}\right) e\right] e .
$$

This identity together with the previous lemmas gives us the necessary machinery to parallel the methods of [6], and in the next section

${ }^{3}$ It may be noted that the only use made of (1) in this lemma, as well as in much of the rest of the paper, was that in combination with (3) it implied strict powerassociativity and Lemma 2. 
we do so obtaining our Theorems 1 and 2 .

3. Keeping our previous notations, we proceed to consider the ideals in $A$ and $A^{+}$generated by the set $A_{e}(1)+A_{e}(0)$. Defining $N$ as the set of all sums of elements of the form $\left[z_{1} y_{1}\right]_{2}$ and $\left[z_{1} s_{0}\right]_{2}$ we will show that both these ideals are precisely the set $N+A_{e}(1)$ $+A_{e}(0)$.

First we observe that a substitution of $s_{0}$ for $y$ in (6) with $\lambda=1$ yields $\left[x_{1} s_{0}\right]_{2}=\left[s_{0} x_{1}\right]_{2}$ so that $N$ contains all terms of the form $\left[s_{0} x_{1}\right]_{2}$ as well.

Lemma 5. $N$ is contained in the ideal in $A^{+}$generated by $A_{e}(1)$ $+A_{e}(0)$, and in particular $\left[y_{1} x_{1}\right]_{2}=\left[e y_{1} * x_{1}\right]_{2}$.

Proof. We show that $2\left[z_{1} x_{0}\right]_{2}=\left[z_{1} * x_{0}\right]_{2}$ and $\left[y_{1} x_{1}\right]_{2}=\left[e y_{1} * x_{1}\right]_{2}$. The $A_{e}(2)$ component of the right member of (9) is just $\left[z_{1} * x_{0}\right]_{2}$ since $\left[\left(z_{1} * x_{0}\right) * e\right]_{2}=\left[z_{1} * x_{0}\right]_{2} * e=2\left[z_{1} * x_{0}\right]_{2}$ and $\left[\left(\left(z_{1} * x_{0}\right) e\right) e\right]_{2}$ $=\left(\left[z_{1} * x_{0}\right]_{2} e\right) e=\left[z_{1} * x_{0}\right]_{2}$. But the $A_{e}(2)$ component of the left member is $2\left[z_{1} x_{0}\right]_{2}$ which is the first desired relation. Now let $\lambda=1$ and $y=y_{1}$ in (6) obtaining $x_{1}\left(y_{1} e\right)+e\left(y_{1} x_{1}\right)-\left(x_{1} y_{1}\right) e=\left(e y_{1}\right) x_{1}$, so that $x_{1} y_{1}+e\left(y_{1} x_{1}\right)-\left(x_{1} y_{1}\right) e=e y_{1} * x_{1}$, and thus $\left[y_{1} x_{1}\right]_{2}=\left[e y_{1} * x_{1}\right]_{2}$.

Lemma 6. The ideal in $A$ generated by $A_{e}(1)+A_{e}(0)$ is just the set $N+A_{e}(1)+A_{e}(0)$.

Proof. We obviously need only show containment of the ideal in the set. By the definition of $N$ and by Lemmas 3 and 4 we have $A \cdot A_{e}(0), A_{e}(0) \cdot A, A \cdot A_{e}(1)$ and $A_{e}(1) \cdot A$ are all in $N+A_{e}(1)+A_{e}(0)$. It remains to show that $A_{e}(2) \cdot N$, and $N \cdot A_{e}(2)$ are contained in $N+A_{e}(1)+A_{e}(0)$. We complete this task, and thus the proof, in the four following cases.

CAse 1 , To SHow $x_{2}\left[y_{1} z_{1}\right]_{2} \in N+A_{\epsilon}(1)+A_{e}(0)$. We follow the proof of [6, Lemma 3.3] to linearize $x^{3} x=x^{2} x^{2}$, consider $A_{e}(2)$ components and obtain $2\left[x_{2}\left(y_{1} * z_{1}\right)\right]_{2}-\left[\left(y_{1} * z_{1}\right) x_{2}\right]_{2} \in N$. Then we linearize (3) to find $\left[x_{2}, y_{1} * z_{1}\right]+\left[y_{1}, x_{2} * z_{1}\right]+\left[z_{1}, x_{2} * y_{1}\right]=0$. Thus $\left[x_{2}\left(y_{1} * z_{1}\right)\right]_{2}$ $-\left[\left(y_{1} * z_{1}\right) x_{2}\right]_{2} \in N$, so we have $\left[x_{2}\left(y_{1} * z_{1}\right)\right]_{2} \in N$, and substituting $e y_{1}$ for $y_{1}$ this becomes $\left[x_{2}\left(e y_{1} * z_{1}\right)\right]_{2} \in N$, but this is just $\left[x_{2}\left[e y_{1} * z_{1}\right]_{2}\right]_{2}$ $\in N$. Now by Lemma $5 x_{2}\left[y_{1} z_{1}\right]_{2}=x_{2}\left[e y_{1} * z_{1}\right]_{2} \in N+A_{e}(1)+A_{e}(0)$.

Case 2, to show $\left[y_{1} z_{1}\right]_{2} x_{2} \in N+A_{e}(1)+A_{e}(0)$. We proceed as in Case 1 except that from the relations $\left[x_{2}\left(y_{1} * z_{1}\right)_{2} \in N\right.$ and $\left[x_{2}\left(y_{1} * z_{1}\right)\right]_{2}$ $-\left[\left(y_{1} * z_{1}\right) x_{2}\right]_{2} \in N$ we now derive $\left[\left(y_{1} * z_{1}\right) x_{2}\right]_{2} \in N$.

CASE 3, TO SHOW $x_{2}\left[y_{1} s_{0}\right]_{2} \in N+A_{e}(1)+A_{e}(0)$. Let $w=e, x=x_{2}$, $y=y_{1}$, and $z=s_{0}$ in (5) to obtain $\left(x_{2} y_{1}\right) s_{0}=x_{2}\left(y_{1} s_{0}\right)$. The $A_{e}(2)$ component of the left member is in $N$ by Lemmas 3 and 4 , and thus $\left[x_{2}\left[y_{1} s_{0}\right]_{2}\right]_{2} \in N$ so that $x_{2}\left[y_{1} s_{0}\right]_{2} \in N+A_{e}(1)+A_{e}(0)$. 
CASE 4 , To show $\left[y_{1} s_{0}\right]_{2} x_{2} \in N+A_{e}(1)+A_{e}(0)$. Since we have Case 3 , it is certainly enough to show $\left[y_{1} s_{0}\right]_{2} * x_{2} \in N$. In $A^{+}$we have [3, Identity 8$]^{4}$ which is just $\left[y_{1} * s_{0}\right]_{2} * x_{2}=2\left[\left[y_{1} * x_{2}\right]_{1} * s_{0}\right]_{2}$, and the right member is in $N$. Since $\left[y_{1} s_{0}\right]_{2}=\left[s_{0} y_{1}\right]_{2}$ as observed earlier, we have the desired relation, $\left[y_{1} s_{0}\right]_{2} * x_{2}=2^{-1}\left[y_{1} * s_{0}\right]_{2} * x_{2} \in N$, and complete the proof of the lemma.

Thus by Lemmas 5 and 6 we see that the ideal in $A$ generated by $A_{e}(1)+A_{e}(0)$ is contained in the ideal in $A^{+}$generated by the same set.

We now suppose that $A$ is semi-simple. As such it will contain an idempotent. Adding the assumption that $A$ is finite dimensional allows us to infer the existence of a principal idempotent $e$. We observe that $e / 2$ is then a principal idempotent of $A^{+}$, and thus by [3, Theorem 7] $A_{e}(1)+A_{e}(0) \subset \operatorname{Rad} A^{+}$. Therefore, $A_{e}(1)+A_{e}(0)$ generates a nil ideal in $A$ so that $A_{e}(1)=A_{e}(0)=0$ by semi-simplicity, and $e$ is an identity element for $A$. The observation in [7] that in the presence of an identity element (1) yields (2), together with [6, Theorem 3.5], completes the proof of Theorem 1. Theorem 2 is a direct application of [6, Theorem 4.2].

\section{BIBLIOGRAPHY}

1. A. A. Albert, On the power-associativity of rings, Summa Brasil. Math. vol. 2 (1948) pp. 21-32.

2. - Power-associative rings, Trans. Amer. Math. Soc. vol. 64 (1948) pp. $552-593$.

3. - A theory of power-associative commutative algebras, Trans. Amer. Math. Soc. vol. 69 (1950) pp. 503-527.

4. L. A. Kokoris, New results on power-associative algebras, Trans. Amer. Math. Soc. vol. 77 (1954) pp. 363-373.

5. J. D. Leadley and R. W. Ritchie, Conditions for the power-associativity of algebras, to appear in Proc. Amer. Math. Soc.

6. R. H. Oehmke, On flexible algebras, Ann. of Math. vol. 68 (1958) pp. 221-230.

7. R. D. Schafer, Noncommutative Jordan algebras of characteristic 0, Proc. Amer. Math. Soc. vol. 6 (1955) pp. 472-475.

Princeton University

${ }^{4}$ A reference to [3] always implies a reference to the corresponding result in [4] for the characteristic 5 case. 